\title{
A CONTINUOUS SPECTRUM FOR NONHOMOGENEOUS DIFFERENTIAL OPERATORS IN ORLICZ-SOBOLEV SPACES
}

\author{
MIHAI MIHĂILESCU and VICENŢIU RĂDULESCU
}

\begin{abstract}
We study the nonlinear eigenvalue problem $-\operatorname{div}(a(|\nabla u|) \nabla u)=\lambda|u|^{q(x)-2} u$ in $\Omega, u=0$ on $\partial \Omega$, where $\Omega$ is a bounded open set in $\mathrm{R}^{N}$ with smooth boundary, $q$ is a continuous function, and $a$ is a nonhomogeneous potential. We establish sufficient conditions on $a$ and $q$ such that the above nonhomogeneous quasilinear problem has continuous families of eigenvalues. The proofs rely on elementary variational arguments. The abstract results of this paper are illustrated by the cases $a(t)=t^{p-2} \log \left(1+t^{r}\right)$ and $a(t)=t^{p-2}[\log (1+t)]^{-1}$.
\end{abstract}

\section{Introduction and preliminary results}

Let $\Omega$ be a bounded domain in $\mathrm{R}^{N}(N \geq 3)$ with smooth boundary $\partial \Omega$. In this paper we are concerned with the following eigenvalue problem:

$$
\begin{cases}-\operatorname{div}(a(|\nabla u|) \nabla u)=\lambda|u|^{q(x)-2} u, & \text { for } x \in \Omega \\ u=0, & \text { for } x \in \partial \Omega .\end{cases}
$$

We assume that the function $a:(0, \infty) \rightarrow \mathrm{R}$ is such that the mapping $\phi:$ $\mathrm{R} \rightarrow \mathrm{R}$ defined by

$$
\phi(t)= \begin{cases}a(|t|) t, & \text { for } t \neq 0 \\ 0, & \text { for } t=0,\end{cases}
$$

is an odd, increasing homeomorphism from $R$ onto $R$. We also suppose throughout this paper that $\lambda>0$ and $q: \bar{\Omega} \rightarrow(1, \infty)$ is a continuous function.

Since the operator in the divergence form is nonhomogeneous we introduce an Orlicz-Sobolev space setting for problems of this type. On the other hand, the term arising in the right hand side of equation (1) is also nonhomogeneous and its particular form appeals to a suitable variable exponent Lebesgue space setting.

We point out that eigenvalue problems involving quasilinear nonhomogeneous problems in Orlicz-Sobolev spaces were studied in [12] but in a different 
framework. In what concerns the case when $a(|\nabla u|)=|\nabla u|^{q(x)-2}$, problem (1) was studied by Fan et al. in [10], [11] who established the existence of a sequence of eigenvalues, by means of the Ljusternik-Schnirelmann critical point theory. Denoting by $\Lambda$ the set of all nonnegative eigenvalues, Fan, Zhang and Zhao showed that sup $\Lambda=+\infty$ and they pointed out that only under additional assumptions we have inf $\Lambda>0$. We remark that in the homogeneous case corresponding the $p$-Laplace operator (that is, if $q(x) \equiv p$ ) we always have inf $\Lambda>0$. A different approach of the eigenvalue problem (1) corresponding to $a(|\nabla u|)=|\nabla u|^{p(x)-2}$ and $p(x) \neq q(x)$ is given in Mihăilescu and Rădulescu [19].

We first recall some basic facts about Orlicz spaces. Define

$$
\Phi(t)=\int_{0}^{t} \phi(s) d s, \quad \Phi^{\star}(t)=\int_{0}^{t} \phi^{-1}(s) d s, \quad \text { for all } \quad t \in \mathrm{R} .
$$

We observe that $\Phi$ is a Young function, that is, $\Phi(0)=0, \Phi$ is convex, and $\lim _{x \rightarrow \infty} \Phi(x)=+\infty$. Furthermore, since $\Phi(x)=0$ if and only if $x=0$, $\lim _{x \rightarrow 0} \Phi(x) / x=0$, and $\lim _{x \rightarrow \infty} \Phi(x) / x=+\infty$, then $\Phi$ is called an $N$ function. The function $\Phi^{\star}$ is called the complementary function of $\Phi$ and it satisfies

$$
\Phi^{\star}(t)=\sup \{s t-\Phi(s) ; s \geq 0\}, \quad \text { for all } t \geq 0 .
$$

We observe that $\Phi^{\star}$ is also an $N$-function and the following Young's inequality holds true:

$$
s t \leq \Phi(s)+\Phi^{\star}(t), \quad \text { for all } s, t \geq 0 .
$$

The Orlicz space $L_{\Phi}(\Omega)$ defined by the $N$-function $\Phi$ (see [2], [3], [4]) is the space of measurable functions $u: \Omega \rightarrow \mathrm{R}$ such that

$$
\|u\|_{L_{\Phi}}:=\sup \left\{\int_{\Omega} u v d x ; \int_{\Omega} \Phi^{\star}(|v|) d x \leq 1\right\}<\infty .
$$

Then $\left(L_{\Phi}(\Omega),\|\cdot\|_{L_{\Phi}}\right)$ is a Banach space whose norm is equivalent to the Luxemburg norm

$$
\|u\|_{\Phi}:=\inf \left\{k>0 ; \int_{\Omega} \Phi\left(\frac{u(x)}{k}\right) d x \leq 1\right\} .
$$

For Orlicz spaces the Hölder's inequality reads as follows (see [21, Inequality 4 , p. 79]):

$$
\int_{\Omega} u v d x \leq 2\|u\|_{L_{\Phi}}\|v\|_{L_{\Phi^{\star}}} \quad \text { for all } u \in L_{\Phi}(\Omega) \text { and } v \in L_{\Phi^{\star}}(\Omega) .
$$


We denote by $W_{0}^{1} L_{\Phi}(\Omega)$ the corresponding Orlicz-Sobolev space for problem (1), equipped with the norm

$$
\|u\|=\||\nabla u|\|_{\Phi}
$$

(see [3], [4], [13]). The space $W_{0}^{1} L_{\Phi}(\Omega)$ is also a Banach space.

In this paper we assume that $\Phi$ and $\Phi^{\star}$ satisfy the $\Delta_{2}$-condition (at infinity), namely

$$
1<\liminf _{t \rightarrow \infty} \frac{t \phi(t)}{\Phi(t)} \leq \limsup _{t>0} \frac{t \phi(t)}{\Phi(t)}<\infty .
$$

Then $L_{\Phi}(\Omega)$ and $W_{0}^{1} L_{\Phi}(\Omega)$ are reflexive Banach spaces.

Now we introduce the Orlicz-Sobolev conjugate $\Phi_{\star}$ of $\Phi$, defined as

$$
\Phi_{\star}^{-1}(t)=\int_{0}^{t} \frac{\Phi^{-1}(s)}{S^{(N+1) / N}} d s
$$

We assume that

(2) $\lim _{t \rightarrow 0} \int_{t}^{1} \frac{\Phi^{-1}(s)}{s^{(N+1) / N}} d s<\infty, \quad$ and $\quad \lim _{t \rightarrow \infty} \int_{1}^{t} \frac{\Phi^{-1}(s)}{s^{(N+1) / N}} d s=\infty$.

Finally, we define

$$
p_{0}:=\inf _{t>0} \frac{t \phi(t)}{\Phi(t)} \quad \text { and } \quad p^{0}:=\sup _{t>0} \frac{t \phi(t)}{\Phi(t)} .
$$

Next, we recall some background facts concerning the variable exponent Lebesgue spaces. For more details we refer to the book by Musielak [20] and the papers by Acerbi et al. [1], Edmunds et al. [6], [7], [8], Kovacik and Rákosník [15], Mihăilescu and Rădulescu [16], Samko and Vakulov [22], Zhikov [24].

Set

$$
C_{+}(\bar{\Omega})=\{h ; h \in C(\bar{\Omega}), h(x)>1 \text { for all } x \in \bar{\Omega}\} .
$$

For any $h \in C_{+}(\bar{\Omega})$ we define

$$
h^{+}=\sup _{x \in \Omega} h(x) \quad \text { and } \quad h^{-}=\inf _{x \in \Omega} h(x) .
$$

For any $q(x) \in C_{+}(\bar{\Omega})$ we define the variable exponent Lebesgue space $L^{q(x)}(\Omega)$ (see [15]). On $L^{q(x)}(\Omega)$ we define the Luxemburg norm by the formula

$$
|u|_{q(x)}=\inf \left\{\mu>0 ; \int_{\Omega}\left|\frac{u(x)}{\mu}\right|^{q(x)} d x \leq 1\right\} .
$$


We remember that the variable exponent Lebesgue spaces are separable and reflexive Banach spaces. If $0<|\Omega|<\infty$ and $q_{1}, q_{2}$ are variable exponents so that $q_{1}(x) \leq q_{2}(x)$ almost everywhere in $\Omega$ then there exists the continuous embedding $L^{q_{2}(x)}(\Omega) \hookrightarrow L^{q_{1}(x)}(\Omega)$.

If $\left(u_{n}\right), u \in L^{q(x)}(\Omega)$ then the following relations hold true

$$
\begin{aligned}
|u|_{q(x)}>1 & \Rightarrow|u|_{q(x)}^{q^{-}} \leq \int_{\Omega}|u|^{q(x)} d x \leq|u|_{q(x)}^{q^{+}} \\
|u|_{q(x)}<1 & \Rightarrow|u|_{q(x)}^{q^{+}} \leq \int_{\Omega}|u|^{q(x)} d x \leq|u|_{q(x)}^{q^{-}} \\
\left|u_{n}-u\right|_{q(x)} \rightarrow 0 & \Leftrightarrow \int_{\Omega}\left|u_{n}-u\right|^{q(x)} d x \rightarrow 0 .
\end{aligned}
$$

\section{The main results}

We say that $\lambda \in \mathrm{R}$ is an eigenvalue of problem (1) if there exists $u \in W_{0}^{1} L_{\Phi}(\Omega) \backslash$ $\{0\}$ such that

$$
\int_{\Omega} a(|\nabla u|) \nabla u \nabla v d x-\lambda \int_{\Omega}|u|^{q(x)-2} u v d x=0,
$$

for all $v \in W_{0}^{1} L_{\Phi}(\Omega)$. We point out that if $\lambda$ is an eigenvalue of problem (1) then the corresponding $u \in W_{0}^{1} L_{\Phi}(\Omega) \backslash\{0\}$ is a weak solution of (1), called an eigenvector of equation (1) corresponding to the eigenvalue $\lambda$.

Our first main result shows that, in certain circumstances, any positive and sufficiently small $\lambda$ is an eigenvalue of (1).

THEOREM 1. Assume that relation (2) is fulfilled and furthermore

$$
1<\inf _{x \in \Omega} q(x)<p_{0},
$$

and

$$
\lim _{t \rightarrow \infty} \frac{|t|^{q^{+}}}{\Phi_{\star}(k t)}=0, \quad \text { for all } k>0 .
$$

Then there exists $\lambda^{\star}>0$ such that any $\lambda \in\left(0, \lambda^{\star}\right)$ is an eigenvalue for problem (1).

The above result implies

$$
\inf _{u \in W_{0}^{1} L_{\Phi}(\Omega) \backslash\{0\}} \frac{\int_{\Omega} \Phi(|\nabla u|) d x}{\int_{\Omega}|u|^{q(x)} d x}=0 .
$$


The second main result of this paper asserts that in certain cases the set of eigenvalues may coincide with the whole positive semi-axis.

THEOREм 2. Assume that relations (2) and (7) are fulfilled and furthermore

$$
\sup _{x \in \Omega} q(x)<p_{0} .
$$

Then every $\lambda>0$ is an eigenvalue for problem (1). Moreover, for any $\lambda>0$ there exists a sequence of eigenvectors $\left\{u_{n}\right\} \subset E$ such that $\lim _{n \rightarrow \infty} u_{n}=0$ in $W_{0}^{1} L_{\Phi}(\Omega)$.

Remark 3. Relations (2) and (7) enable us to apply Theorem 2.2 in [12] (see also Theorem 8.33 in [3]) in order to obtain that $W_{0}^{1} L_{\Phi}(\Omega)$ is compactly embedded in $L^{q^{+}}(\Omega)$. This fact combined with the continuous embedding of $L^{q^{+}}(\Omega)$ in $L^{q(x)}(\Omega)$ ensures that $W_{0}^{1} L_{\Phi}(\Omega)$ is compactly embedded in $L^{q(x)}(\Omega)$.

REMARK 4. The conclusion of Theorems 1 and 2 still remains valid if we replace the hypothesis (7) in Theorems 1 and 2 by the following relation

$$
N<p_{0}<\liminf _{t \rightarrow \infty} \frac{\log (\Phi(t))}{\log (t)}
$$

Indeed, using Lemma D.2 in [5], it follows that $W_{0}^{1} L_{\Phi}(\Omega)$ is continuously embedded in $W_{0}^{1, p_{0}}(\Omega)$. On the other hand, since we assume $p_{0}>N$, we deduce that $W_{0}^{1, p_{0}}(\Omega)$ is compactly embedded in $C(\bar{\Omega})$. Thus, we obtain that $W_{0}^{1} L_{\Phi}(\Omega)$ is compactly embedded in $C(\bar{\Omega})$. Since $\Omega$ is bounded it follows that $W_{0}^{1} L_{\Phi}(\Omega)$ is continuously embedded in $L^{q(x)}(\Omega)$.

\section{Proof of Theorem 1}

Let $E$ denote the Orlicz-Sobolev space $W_{0}^{1} L_{\Phi}(\Omega)$.

For any $\lambda>0$ the energy functional $J_{\lambda}: E \rightarrow \mathrm{R}$ corresponding to problem (1) is defined by

$$
J_{\lambda}(u)=\int_{\Omega} \Phi(|\nabla u|) d x-\lambda \int_{\Omega} \frac{1}{q(x)}|u|^{q(x)} d x .
$$

Standard arguments imply that $J_{\lambda} \in C^{1}(E, \mathrm{R})$ and

$$
\left\langle J_{\lambda}^{\prime}(u), v\right\rangle=\int_{\Omega} a(|\nabla u|) \nabla u \nabla v d x-\lambda \int_{\Omega}|u|^{q(x)-2} u v d x,
$$


for all $u, v \in E$. Thus the weak solutions of (1) coincide with the critical points of $J_{\lambda}$. If such a weak solution exists and is nontrivial then the corresponding $\lambda$ is an eigenvalue of problem (1).

Lemma 5. There is some $\lambda^{\star}>0$ such that for any $\lambda \in\left(0, \lambda^{\star}\right)$ there exist $\rho, \alpha>0$ such that $J_{\lambda}(u) \geq \alpha>0$ for any $u \in E$ with $\|u\|=\rho$.

Proof. By the definition of $p^{0}$ and since $\frac{\mathrm{d}}{\mathrm{d} \tau}\left(\tau^{p_{0}} \Phi(t / \tau)\right) \geq 0$ we obtain

$$
\Phi(t) \geq \tau^{p^{0}} \Phi(t / \tau), \quad \forall t>0 \text { and } \tau \in(0,1],
$$

(see page 44 in [4]). Combining this fact with Proposition 6 in [21, page 77] we find that

$$
\int_{\Omega} \Phi(|\nabla u(x)|) d x \geq\|u\|^{p^{0}}, \quad \forall u \in E \text { with }\|u\|<1 .
$$

On the other hand, since $E$ is continuously embedded in $L^{q(x)}(\Omega)$, there exists a positive constant $c_{1}$ such that

$$
|u|_{q(x)} \leq c_{1}\|u\|, \quad \forall u \in E .
$$

We fix $\rho \in(0,1)$ such that $\rho<1 / c_{1}$. Then relation (11) implies

$$
|u|_{q(x)}<1, \quad \forall u \in E, \text { with }\|u\|=\rho .
$$

Furthermore, relation (4) yields

$$
\int_{\Omega}|u|^{q(x)} d x \leq|u|_{q(x)}^{q^{-}}, \quad \forall u \in E, \text { with }\|u\|=\rho .
$$

Relations (11) and (13) imply

$$
\int_{\Omega}|u|^{q(x)} d x \leq c_{1}^{q^{-}}\|u\|^{q^{-}}, \quad \forall u \in E, \text { with }\|u\|=\rho .
$$

Taking into account relations (10), (4) and (14) we deduce that for any $u \in E$ with $\|u\|=\rho$ the following inequalities hold true

$$
J_{\lambda}(u) \geq\|u\|^{p^{0}}-\frac{\lambda}{q^{-}} \int_{\Omega}|u|^{q(x)} d x=\rho^{q^{-}}\left(\rho^{p^{0}-q^{-}}-\frac{\lambda}{q^{-}} c_{1}^{q^{-}}\right) .
$$

We point out that by relation (6) and the definition of $p^{0}$ we have $q^{-}<l \leq p^{0}$. By the above inequality we remark that if we define

$$
\lambda^{\star}=\frac{\rho^{p^{0}-q^{-}}}{2} \cdot \frac{q^{-}}{c_{1}^{q^{-}}}
$$


then for any $\lambda \in\left(0, \lambda^{\star}\right)$ and any $u \in E$ with $\|u\|=\rho$ there exists $\alpha=\frac{\rho^{p^{0}}}{2}>0$ such that

$$
J_{\lambda}(u) \geq \alpha>0 .
$$

The proof of Lemma 5 is complete.

Lemma 6. There exists $\phi \in E$ such that $\phi \geq 0, \varphi \neq 0$ and $J_{\lambda}(t \phi)<0$, for $t>0$ small enough.

Proof. Assumption (6) implies that $q^{-}<p_{0}$. Let $\epsilon_{0}>0$ be such that $q^{-}+\epsilon_{0}<p_{0}$. On the other hand, since $q \in C(\bar{\Omega})$ it follows that there exists an open set $\Omega_{0} \subset \Omega$ such that $\left|q(x)-q^{-}\right|<\epsilon_{0}$ for all $x \in \Omega_{0}$. Thus, we conclude that $q(x) \leq q^{-}+\epsilon_{0}<p_{0}$ for all $x \in \Omega_{0}$.

Let $\phi \in C_{0}^{\infty}(\Omega)$ be such that $\operatorname{supp}(\phi) \supset \bar{\Omega}_{0}, \phi(x)=1$ for all $x \in \bar{\Omega}_{0}$ and $0 \leq \phi \leq 1$ in $\Omega$.

We also point out that there exists $t_{0} \in(0,1)$ such that for any $t \in\left(0, t_{0}\right)$ we have

$$
\|t|\nabla \phi|\|_{\Phi}=t\|\phi\|<1 .
$$

Taking into account all the above information and using Lemma C.9 in [5] we have

$$
\begin{aligned}
J_{\lambda}(t \phi) & =\int_{\Omega} \Phi(t|\nabla \phi(x)|) d x-\lambda \int_{\Omega} \frac{t^{q(x)}}{q(x)}|\phi|^{q(x)} d x \\
& \leq \int_{\Omega} \Phi(t|\nabla \phi(x)|) d x-\frac{\lambda}{q^{+}} \int_{\Omega} t^{q(x)}|\phi|^{q(x)} d x \\
& \leq \int_{\Omega} \Phi(t|\nabla \phi(x)|) d x-\frac{\lambda}{q^{+}} \int_{\Omega_{0}} t^{q(x)}|\phi|^{q(x)} d x \\
& \leq t^{p_{0}}\|\phi\|^{p_{0}}-\frac{\lambda \cdot t^{q^{-}+\epsilon_{0}}}{q^{+}}\left|\Omega_{0}\right|,
\end{aligned}
$$

for any $t \in(0,1)$, where $\left|\Omega_{0}\right|$ denotes the Lebesgue measure of $\Omega_{0}$. Therefore

$$
J_{\lambda}(t \phi)<0
$$

for $t<\delta^{1 /\left(p_{0}-q^{-}-\epsilon_{0}\right)}$, where

$$
0<\delta<\min \left\{t_{0}, \frac{\frac{\lambda}{q^{+}}\left|\Omega_{0}\right|}{\|\phi\|^{p_{0}}}\right\} .
$$

The proof of Lemma 6 is complete. 
Proof of Theorem 1. Let $\lambda^{\star}>0$ be defined as in (15) and $\lambda \in\left(0, \lambda^{\star}\right)$. By Lemma 5 it follows that on the boundary of the ball centered at the origin and of radius $\rho$ in $E$, denoted by $B_{\rho}(0)$, we have

$$
\inf _{\partial B_{\rho}(0)} J_{\lambda}>0 .
$$

On the other hand, by Lemma 6 , there exists $\phi \in E$ such that $J_{\lambda}(t \phi)<0$ for all $t>0$ small enough. Moreover, relations (10), (14) and (4) imply that for any $u \in B_{\rho}(0)$ we have

$$
J_{\lambda}(u) \geq\|u\|^{p^{0}}-\frac{\lambda}{q^{-}} c_{1}^{q^{-}}\|u\|^{q^{-}} .
$$

It follows that

$$
-\infty<\underline{c}:=\frac{\inf }{B_{\rho}(0)} J_{\lambda}<0 .
$$

We let now $0<\epsilon<\inf _{\partial B_{\rho}(0)} J_{\lambda}-\inf _{B_{\rho}(0)} J_{\lambda}$. Applying Ekeland's variational principle [9] to the functional $J_{\lambda}: \overline{B_{\rho}(0)} \rightarrow \mathrm{R}$, we find $u_{\epsilon} \in \overline{B_{\rho}(0)}$ such that

$$
\begin{aligned}
& J_{\lambda}\left(u_{\epsilon}\right)<\inf _{B_{\rho}(0)} J_{\lambda}+\epsilon \\
& J_{\lambda}\left(u_{\epsilon}\right)<J_{\lambda}(u)+\epsilon \cdot\left\|u-u_{\epsilon}\right\|, \quad u \neq u_{\epsilon} .
\end{aligned}
$$

Since

$$
J_{\lambda}\left(u_{\epsilon}\right) \leq \inf _{B_{\rho}(0)} J_{\lambda}+\epsilon \leq \inf _{B_{\rho}(0)} J_{\lambda}+\epsilon<\inf _{\partial B_{\rho}(0)} J_{\lambda}
$$

we deduce that $u_{\epsilon} \in B_{\rho}(0)$. Now, we define $I_{\lambda}: \overline{B_{\rho}(0)} \rightarrow \mathrm{R}$ by $I_{\lambda}(u)=$ $J_{\lambda}(u)+\epsilon \cdot\left\|u-u_{\epsilon}\right\|$. It is clear that $u_{\epsilon}$ is a minimum point of $I_{\lambda}$ and thus

$$
\frac{I_{\lambda}\left(u_{\epsilon}+t \cdot v\right)-I_{\lambda}\left(u_{\epsilon}\right)}{t} \geq 0
$$

for small $t>0$ and any $v \in B_{1}(0)$. The above relation yields

$$
\frac{J_{\lambda}\left(u_{\epsilon}+t \cdot v\right)-J_{\lambda}\left(u_{\epsilon}\right)}{t}+\epsilon \cdot\|v\| \geq 0 .
$$

Letting $t \rightarrow 0$ it follows that $\left\langle J_{\lambda}^{\prime}\left(u_{\epsilon}\right), v\right\rangle+\epsilon \cdot\|v\|>0$ and we infer that $\left\|J_{\lambda}^{\prime}\left(u_{\epsilon}\right)\right\| \leq \epsilon$.

We deduce that there exists a sequence $\left\{w_{n}\right\} \subset B_{\rho}(0)$ such that

$$
J_{\lambda}\left(w_{n}\right) \rightarrow \underline{c} \quad \text { and } \quad J_{\lambda}^{\prime}\left(w_{n}\right) \rightarrow 0 .
$$


It is clear that $\left\{w_{n}\right\}$ is bounded in $E$. Thus, there exists $w \in E$ such that, up to a subsequence, $\left\{w_{n}\right\}$ converges weakly to $w$ in $E$. By Remark 4 we deduce that $E$ is compactly embeddded in $L^{q(x)}(\Omega)$, hence $\left\{w_{n}\right\}$ converges strongly to $w$ in $L^{q(x)}(\Omega)$. So, by relations (5) and Hölder's inequality for variable exponent spaces (see e.g. [15]),

$$
\lim _{n \rightarrow \infty} \int_{\Omega}\left|w_{n}\right|^{q(x)} d x=\int_{\Omega}|w|^{q(x)} d x
$$

and

$$
\lim _{n \rightarrow \infty} \int_{\Omega}\left|w_{n}\right|^{q(x)-2} w_{n} v d x=\int_{\Omega}|w|^{q(x)-2} w v d x
$$

for any $v \in E$.

We conclude that $w$ is a nontrivial weak solution for problem (1) and thus any $\lambda \in\left(0, \lambda^{\star}\right)$ is an eigenvalue of problem (1). Similar arguments as those used on page 50 in [4] imply that $\left\{w_{n}\right\}$ converges strongly to $w$ in $E$. So, by (17),

$$
J_{\lambda}(w)=\underline{c}<0 \quad \text { and } \quad J_{\lambda}^{\prime}(w)=0 .
$$

The proof of Theorem 1 is complete.

\section{Proof of Theorem 2}

We still denote by $E$ the Orlicz-Sobolev space $W_{0}^{1} L_{\Phi}(\Omega)$. For any $\lambda>0$ let $J_{\lambda}$ be defined as in the above section of the paper.

In order to prove Theorem 2 we apply to the functional $J_{\lambda}$ a symmetric version of the mountain pass lemma, recently developed by Kajikia in [14]. Before presenting the result in [14] we remember the following definition.

Definition 7. Let $X$ be a real Banach space. We say that a subset $A$ of $X$ is symmetric if $u \in A$ implies $-u \in A$. For a closed symmetric set $A$ which does not contain the origin, we define the genus $\gamma(A)$ of $A$ as the smallest integer $k$ such that there exists an odd continuous mapping from $A$ to $\mathbf{R}^{k} \backslash\{0\}$. If there does not exist such an integer $k$, we define $\gamma(A)=+\infty$. Moreover, we set $\gamma(\emptyset)=0$. Finally, we denote by $\Gamma_{k}$ the family

$$
\Gamma_{k}=\{A \subset X ; 0 \notin A \text { and } \gamma(A) \geq k\} .
$$

We state now the symmetric mountain pass lemma of Kajikia (see Theorem 1 in [14]).

Theorem 8. Assume $X$ is an infinite dimensional Banach space and $\Lambda \in$ $C^{1}(X, \mathrm{R})$ satisfies conditions (A1) and (A2) below. 
(A1) $\Lambda(u)$ is even, bounded from below, $\Lambda(0)=0$ and $\Lambda(u)$ satisfies the Palais-Smale condition (i.e., any sequence $\left\{u_{n}\right\}$ in $X$ such that $\left\{\Lambda\left(u_{n}\right)\right\}$ is bounded and $\Lambda^{\prime}\left(u_{n}\right) \rightarrow 0$ in $X^{\star}$ as $n \rightarrow \infty$ has a convergent subsequence);

(A2) For each $k \in \mathrm{N}$, there exists an $A_{k} \in \Gamma_{k}$ such that $\sup _{u \in A_{k}} \Lambda(u)<0$.

Under the above assumptions, either (i) or (ii) below holds true.

(i) There exists a sequence $\left\{u_{n}\right\}$ such that $\Lambda^{\prime}\left(u_{n}\right)=0, \Lambda\left(u_{n}\right)<0$ and $\left\{u_{n}\right\}$ converges to zero;

(ii) There exist two sequences $\left\{u_{n}\right\}$ and $\left\{v_{n}\right\}$ such that $\Lambda^{\prime}\left(u_{n}\right)=0, \Lambda\left(u_{n}\right)=$ $0, u_{n} \neq 0, \lim _{n \rightarrow \infty} u_{n}=0, \Lambda^{\prime}\left(v_{n}\right)=0, \Lambda\left(v_{n}\right)=0$, and $v_{n}$ converges to a non-zero limit.

In order to apply Theorem 8 to the functional $J_{\lambda}$ we prove two auxiliary results.

LEMma 9. The functional $J_{\lambda}$ satisfies condition (A1) from Theorem 8.

Proof. Clearly, $J_{\lambda}(u)=J_{\lambda}(-u)$ for any $u \in E$, i.e. $J_{\lambda}$ is even, and $J_{\lambda}(0)=0$. On the other hand, since by relation (10) we have

$$
\int_{\Omega} \Phi(|\nabla u(x)|) d x \geq\|u\|^{p^{0}}, \quad \forall u \in E \text { with }\|u\|<1,
$$

while by Lemma C.9 in [5] we have

$$
\int_{\Omega} \Phi(|\nabla u(x)|) d x \geq\|u\|^{p_{0}}, \quad \forall u \in E \text { with }\|u\|>1,
$$

we deduce that

$$
\int_{\Omega} \Phi(|\nabla u(x)|) d x \geq \alpha(\|u\|), \quad \forall u \in E,
$$

where $\alpha:[0, \infty) \rightarrow \mathrm{R}, \alpha(t)=t^{p^{0}}$ if $t \leq 1$ and $\alpha(t)=t^{p_{0}}$ if $t>1$.

By Remark 3, the space $E$ is continuously embedded in $L^{q^{ \pm}}(\Omega)$. Thus, there exist two positive constants $d_{1}$ and $d_{2}$ such that

$$
\int_{\Omega}|u|^{q^{+}} d x \leq d_{1}\|u\|^{q^{+}}, \quad \int_{\Omega}|u|^{q^{-}} d x \leq d_{2}\|u\|^{q^{-}}, \quad \forall u \in E .
$$

Combining relations (19) and (20) we get

$$
J_{\lambda}(u) \geq \alpha(\|u\|)-\frac{d_{1} \lambda}{q^{-}}\|u\|^{q^{+}}-\frac{d_{2} \lambda}{q^{-}}\|u\|^{q^{-}}, \quad \forall u \in E .
$$


Since by relation (8) we have $q^{+}<p_{0}$ the above relation shows that $J_{\lambda}$ is bounded from below.

Next, we show that $J_{\lambda}$ satisfies the Palais-Smale condition. Let $\left\{u_{n}\right\}$ be a sequence in $E$ such that $\left\{J_{\lambda}\left(u_{n}\right)\right\}$ is bounded and $J^{\prime}\left(u_{n}\right) \rightarrow 0$ in $E^{\star}$, as $n \rightarrow \infty$. We show that $\left\{u_{n}\right\}$ is bounded in $E$. Assume by contradiction the contrary. Then, passing eventually to a subsequence, still denoted by $\left\{u_{n}\right\}$, we may assume that $\left\|u_{n}\right\| \rightarrow \infty$ as $n \rightarrow \infty$. Thus we may consider that $\left\|u_{n}\right\|>1$ for any integer $n$.

By our assumptions, there is a positive constant $M$ such that for all $n$ large enough we have

$$
\begin{aligned}
M+1+\left\|u_{n}\right\| \geq & J_{\lambda}\left(u_{n}\right)-\frac{1}{q^{-}}\left\langle J^{\prime}\left(u_{n}\right), u_{n}\right\rangle \\
= & \int_{\Omega} \Phi\left(\left|\nabla u_{n}\right|\right) d x-\lambda \int_{\Omega} \frac{1}{q(x)}\left|u_{n}\right|^{q(x)} d x \\
& \quad-\frac{1}{q^{-}} \cdot \int_{\Omega} \phi\left(\left|\nabla u_{n}(x)\right|\right)\left|\nabla u_{n}(x)\right| d x+\frac{\lambda}{q^{-}} \int_{\Omega}\left|u_{n}\right|^{q(x)} d x \\
\geq & \int_{\Omega} \Phi\left(\left|\nabla u_{n}\right|\right) d x-\frac{1}{q^{-}} \cdot \int_{\Omega} \phi\left(\left|\nabla u_{n}(x)\right|\right)\left|\nabla u_{n}(x)\right| d x \\
\geq & \left(1-\frac{p^{0}}{q^{-}}\right) \int_{\Omega} \Phi\left(\left|\nabla u_{n}\right|\right) d x \geq\left(1-\frac{p^{0}}{q^{-}}\right)\left\|u_{n}\right\|^{p_{0}} .
\end{aligned}
$$

Since $p_{0}>1$, letting $n \rightarrow \infty$ we obtain a contradiction. It follows that $\left\{u_{n}\right\}$ is bounded in $E$. Similar arguments as those used in the end of the proof of Theorem 1 imply that, up to a subsequence, $\left\{u_{n}\right\}$ converges strongly in $E$.

The proof of Lemma 9 is complete.

LEMma 10. The functional $J_{\lambda}$ satisfies condition (A2) from Theorem 8.

Proof. We construct a sequence of subsets $A_{k} \in \Gamma_{k}$ such that $\sup _{u \in A_{k}} J_{\lambda}(u)$ $<0$, for each $k \in \mathbf{N}$.

Let $x_{1} \in \Omega$ and $r_{1}>0$ be such that $\overline{B_{r_{1}}\left(x_{1}\right)} \subset \Omega$ and $\left|\overline{B_{r_{1}}\left(x_{1}\right)}\right|<|\Omega| / 2$. Consider $\theta_{1} \in C_{0}^{\infty}(\Omega)$ be a function with $\operatorname{supp}\left(\theta_{1}\right)=\overline{B_{r_{1}}\left(x_{1}\right)}$.

Define $\Omega_{1}=\Omega \backslash \overline{B_{r_{1}}\left(x_{1}\right)}$.

Next, let $x_{2} \in \Omega$ and $r_{2}>0$ be such that $\overline{B_{r_{2}}\left(x_{2}\right)} \subset \Omega_{1}$ and $\left|\overline{B_{r_{2}}\left(x_{2}\right)}\right|<$ $\left|\Omega_{1}\right| / 2$. Consider $\theta_{2} \in C_{0}^{\infty}(\Omega)$ be a function with $\operatorname{supp}\left(\theta_{2}\right)=\overline{B_{r_{2}}\left(x_{2}\right)}$.

Continuing the process described above we can construct by recurrence a sequence of functions $\theta_{1}, \theta_{2}, \ldots, \theta_{k} \in C_{0}^{\infty}(\Omega)$ such that $\operatorname{supp}\left(\theta_{i}\right) \neq \operatorname{supp}\left(\theta_{j}\right)$ if $i \neq j$ and $\left|\operatorname{supp}\left(\theta_{i}\right)\right|>0$ for any $i, j \in\{1, \ldots, k\}$.

We define the finite dimensional subspace of $E$,

$$
F=\operatorname{span}\left\{\theta_{1}, \theta_{2}, \ldots, \theta_{k}\right\}
$$


Clearly, $\operatorname{dim} F=k$ and $\int_{\Omega}|\theta|^{q(x)} d x>0$, for any $\theta \in F \backslash\{0\}$. We denote by $S_{1}$ the unit sphere in $E$, i.e. $S_{1}=\{u \in E ;\|u\|=1\}$. For any number $t \in(0,1)$ we define the set

$$
A_{k}(t)=t \cdot\left(S_{1} \cap F\right)
$$

Since for any bounded symmetric neighborhood $\omega$ of the origin in $\mathrm{R}^{k}$ there holds $\gamma(\partial \omega)=k$ (see Proposition 5.2 in [23]) we deduce that $\gamma\left(A_{k}(t)\right)=k$ for any $t \in(0,1)$.

Finally, we show that for each integer $k$ there exists $t_{k} \in(0,1)$ such that

$$
\sup _{u \in A_{k}\left(t_{k}\right)} J_{\lambda}(u)<0
$$

For any $t \in(0,1)$ we have

$$
\begin{aligned}
\sup _{u \in A_{k}(t)} J_{\lambda}(u) & \leq \sup _{\theta \in S_{1} \cap F} J_{\lambda}(t \theta) \\
& =\sup _{\theta \in S_{1} \cap F}\left\{\int_{\Omega} \Phi(t|\nabla \theta|) d x-\lambda \int_{\Omega} \frac{1}{q(x)} t^{q(x)}|\theta|^{q(x)} d x\right\} \\
& \leq \sup _{\theta \in S_{1} \cap F}\left\{t^{p_{0}} \int_{\Omega} \Phi(|\nabla \theta|) d x-\frac{\lambda t^{q^{+}}}{q^{+}} \int_{\Omega}|\theta|^{q(x)} d x\right\} \\
& =\sup _{\theta \in S_{1} \cap F}\left\{t^{p_{0}}\left(1-\frac{\lambda}{q^{+}} \cdot \frac{1}{t^{p_{0}-q^{+}}} \cdot \int_{\Omega}|\theta|^{q(x)} d x\right)\right\}
\end{aligned}
$$

Since $S_{1} \cap F$ is compact we have $m=\min _{\theta \in S_{1} \cap F} \int_{\Omega}|\theta|^{q(x)} d x>0$. Combining that fact with the information given by relation (8), that is $p_{0}>q^{+}$, we deduce that we can choose $t_{k} \in(0,1)$ small enough such that

$$
1-\frac{\lambda}{q^{+}} \cdot \frac{1}{t^{p_{0}-q^{+}}} \cdot m<0
$$

The above relations yield

$$
\sup _{u \in A_{k}\left(t_{k}\right)} J_{\lambda}(u)<0
$$

The proof of Lemma 10 is complete.

Proof of Theorem 2. Using Lemmas 9 and 10 we deduce that we can apply Theorem 8 to the functional $J_{\lambda}$. So, there exists a sequence $\left\{u_{n}\right\} \subset E$ such that $J^{\prime}\left(u_{n}\right)=0$, for each $n, J_{\lambda}\left(u_{n}\right) \leq 0$ and $\left\{u_{n}\right\}$ converges to zero in $E$.

The proof of Theorem 2 is complete. 


\section{Examples}

In this section we point out two concrete examples of problems to which we can apply the main results of this paper.

Example 1. We consider the problem

$$
\left\{\begin{array}{cl}
-\operatorname{div}\left(\log \left(1+|\nabla u|^{r}\right)|\nabla u|^{p-2} \nabla u\right)=\lambda|u|^{q(x)-2} u, & \text { for } x \in \Omega \\
u=0, & \text { for } x \in \partial \Omega,
\end{array}\right.
$$

where $p$ and $r$ are real numbers such that $1<p, r, N>p+r$ and $q(x)$ is a continuous function on $\bar{\Omega}$ such that $1<q(x)$ for all $x \in \bar{\Omega}$ and furthermore

$$
\inf _{\Omega} q(x)<p \quad \text { and } \quad \sup _{\Omega} q(x)<\frac{N p}{N-p} .
$$

In this case we have

$$
\phi(t)=\log \left(1+|t|^{r}\right) \cdot|t|^{p-2} t, \quad \text { for all } t \in \mathrm{R}
$$

and

$$
\Phi(t)=\int_{0}^{t} \phi(s), \quad \text { for all } \quad t \in \mathrm{R} .
$$

Clearly, $\phi$ is an odd, increasing homeomorphism of $R$ into $R$, while $\Phi$ is convex and even on $R$ and increasing from $R_{+}$to $R_{+}$.

By Example 2 on p. 243 in [5] we know that

$$
p_{0}=p \quad \text { and } \quad p^{0}=p+r
$$

and thus relation (6) in Theorem 1 is satisfied. On the other hand, by Proposition 1 in [18] (see also [17]) we deduce that relations (2) and (7) are fulfilled. Thus, we verified that we can apply Theorem 1 in order to find out that there exists $\lambda^{\star}>0$ such that any $\lambda \in\left(0, \lambda^{\star}\right)$ is an eigenvalue for problem (21).

Example 2. We consider the problem

$$
\left\{\begin{array}{cl}
-\operatorname{div}\left(\frac{|\nabla u|^{p-2} \nabla u}{\log (1+|\nabla u|)}\right)=\lambda|u|^{q(x)-2} u, & \text { for } x \in \Omega \\
u=0, & \text { for } x \in \partial \Omega,
\end{array}\right.
$$

where $p$ is a real number such that $p>N+1$ and $q \in C(\bar{\Omega})$ satisfies $1<q(x)<p-1$ for any $x \in \bar{\Omega}$. In this case we have

$$
\phi(t)=\frac{|t|^{p-2}}{\log (1+|t|)} t
$$


and

$$
\Phi(t)=\int_{0}^{t} \phi(s) d s,
$$

is an increasing continuous function from $\mathrm{R}^{+}$to $\mathrm{R}^{+}$, with $\Phi(0)=0$ and such that the function $\Phi(\sqrt{t})$ is convex. By Example 3 on p. 243 in [5] we have

$$
p_{0}=p-1<p^{0}=p=\liminf _{t \rightarrow \infty} \frac{\log (\Phi(t))}{\log (t)} .
$$

Thus, conditions (2), (8) and (9) from Theorem 2 and Remark 4 are verified. We deduce that every $\lambda>0$ is an eigenvalue for problem (22). Moreover, for each $\lambda>0$ there exists a sequence of eigenvectors $\left\{u_{n}\right\}$ such that $\lim _{n \rightarrow \infty} u_{n}=0$ in $W_{0}^{1} L_{\Phi}(\Omega)$.

ACKNOWLEDGEMENTS. The authors have been supported by Grant CNCSIS PNII-79/2007 "Procese Neliniare Degenerate şi Singulare”. V. Rădulescu has also been supported by Grants CEEX 05-D11-36/2005 and GAR 315/2008.

\section{REFERENCES}

1. Acerbi, E., and Mingione, G., Regularity results for a class of functionals with non-standard growth, Arch. Rational Mech. Anal. 156 (2001), 121-140.

2. Adams, D. R., and Hedberg, L. I., Function Spaces and Potential Theory, Grundlehren Math. Wiss. 314 (1996).

3. Adams, R., Sobolev Spaces, Academic Press, New York, 1975.

4. Clément, Ph., García-Huidobro, M., Manásevich, R., and Schmitt, K., Mountain pass type solutions for quasilinear elliptic equations, Calc. Var. Partial Differential Equations 11 (2000), 33-62.

5. Clément, Ph., de Pagter, B., Sweers, G., and de Thélin, F., Existence of solutions to a semilinear elliptic system through Orlicz-Sobolev spaces, Mediterr. J. Math. 1 no. 3 (2004), 241-267.

6. Edmunds, D. E., Lang, J., and Nekvinda, A., On $L^{p(x)} n o r m s$, Proc. Roy. Soc. London Ser. A 455 (1999), 219-225.

7. Edmunds, D. E., and Rákosník, J., Density of smooth functions in $W^{k, p(x)}(\Omega)$, Proc. Roy. Soc. London Ser. A 437 (1992), 229-236.

8. Edmunds, D. E., and Rákosník, J., Sobolev embedding with variable exponent, Studia Math. 143 (2000), 267-293.

9. Ekeland, I., On the variational principle, J. Math. Anal. Appl. 47 (1974), 324-353.

10. Fan, X., and Zhao, D., A class of De Giorgi type and Hölder continuity, Nonlinear Anal. Ser. A: Theory Methods 36 (1999), 295-318.

11. Fan, X., Zhang, Q., and Zhao, D., Eigenvalues of $p(x)$-Laplacian Dirichlet problem, J. Math. Anal. Appl. 302 (2005), 306-317.

12. Garciá-Huidobro, M., Le, V. K., Manásevich, R., and Schmitt, K., On principal eigenvalues for quasilinear elliptic differential operators: an Orlicz-Sobolev space setting, NoDEA Nonlinear Differential Equations Appl. 6 (1999), 207-225.

13. Gossez, J. P., Nonlinear elliptic boundary value problems for equations with rapidly (or slowly) increasing coefficients, Trans. Amer. Math. Soc. 190 (1974), 163-205. 
14. Kajikia, R., A critical point theorem related to the symmetric mountain pass lemma and its applications to elliptic equations, J. Funct. Anal. 225 (2005), 352-370.

15. Kováčik, O., and Rákosník, J., On spaces $L^{p(x)}$ and $W^{1}, p(x)$, Czechoslovak Math. J. 41 (1991), 592-618.

16. Mihăilescu, M., and Rădulescu, V., A multiplicity result for a nonlinear degenerate problem arising in the theory of electrorheological fluids, Proc. Roy. Soc. London Ser. A 462 (2006), 2625-2641.

17. Mihăilescu, M., and Rădulescu, V., Nonhomogeneous boundary value problems in OrliczSobolev spaces, C. R. Acad. Sci. Paris, Ser. I 344 (2007), 15-20.

18. Mihăilescu, M., and Rădulescu, V., Existence and multiplicity of solutions for quasilinear nonhomogeneous problems: an Orlicz-Sobolev space setting, J. Math. Anal. Appl. 330 (2007), 416-432.

19. Mihăilescu, M., and Rădulescu, V., On a nonhomogeneous quasilinear eigenvalue problem in Sobolev spaces with variable exponent, Proc. Amer. Math. Soc. 135 (2007), 2929-2937.

20. Musielak, J., Orlicz Spaces and Modular Spaces, Lecture Notes in Math. 1034 (1983).

21. Rao, M. M., and Ren, Z. D., Theory of Orlicz Spaces, Marcel Dekker, Inc., New York, 1991.

22. Samko, S., and Vakulov, B., Weighted Sobolev theorem with variable exponent for spatial and spherical potential operators, J. Math. Anal. Appl. 310 (2005), 229-246.

23. Struwe, M., Variational Methods: Applications to Nonlinear Partial Differential Equations and Hamiltonian Systems, Springer-Verlag, Heidelberg, 1996.

24. Zhikov, V. V., On some variational problems, Russian J. Math. Phys. 5 (1997), 105-116.

DEPARTMENT OF MATHEMATICS

UNIVERSITY OF CRAIOVA

200585 CRAIOVA

ROMANIA

E-mail: mmihailes@yahoo.com, vicentiu.radulescu@math.cnrs.fr

INSTITUTE OF MATHEMATICS "SIMION STOILOW" OF

THE ROMANIAN ACADEMY

P.O. BOX 1-764

014700 BUCHAREST

ROMANIA

E-mail: vicentiu.radulescu@math.cnrs.fr
DEPARTMENT OF MATHEMATICS CENTRAL EUROPEAN UNIVERSITY 1051 BUDAPEST

HUNGARY

E-mail: mmihailes@yahoo.com 